\title{
AN INVARIANT OF LINKS IN A HANDLEBODY ASSOCIATED WITH THE SPIN $j$ REPRESENTATION OF $U_{q}(\mathfrak{s l}(2, \mathbb{C}))$
}

\author{
SHIGEKAZU NAKABO
}

(Communicated by Frederick R. Cohen)

\begin{abstract}
We construct an invariant of framed links in a handlebody by means of the spin $j$ representation of $U_{q}(\mathfrak{s l}(2, \mathbb{C}))$. We can see this invariant is an extension of the Jones polynomial and Kauffman's Dubrovnik polynomial. Moreover, we can obtain a linear representation of the generalized braid group associated with the Lie algebras of types $B$ and $C$ by applying the operators used in constructing an invariant to tangles in a solid torus.
\end{abstract}

\section{INTRODUCTION}

In [12] Turaev constructed an invariant of oriented links embedded in a solid torus by means of the vector representation of $U_{q}(\mathfrak{s l}(m, \mathbb{C}))$. We extend his method to links in a handlebody and construct an invariant of framed links in a handlebody by means of the spin $j$ representation of $U_{q}(\mathfrak{s l}(2, \mathbb{C}))$.

Let $D$ be the generic diagram obtained by projecting a framed link $L$ in a handlebody $\Sigma_{n} \times[0,1]$ of genus $n$ onto $\Sigma_{n}$, where $\Sigma_{n}$ is an $n$-holed disk. By a height function on $D$, we associate to each noncritical level a tensor product of some $V_{j}$ 's, where $V_{j}$ is the representation space of the spin $j$ representation of $U_{q}(\mathfrak{s l}(2, \mathbb{C}))$. Then, to each strip including a critical point, we define a linear operator derived from the tensor representation of $U_{q}(\mathfrak{s l}(2, \mathbb{C}))$. Furthermore, we introduce another operator that includes the parameters $\left\{t_{i}^{(l)}\right\}_{i, l}$ depending on the embedding of a link into a handlebody. Let $P_{j}(L)$ denote the image of $1 \in \mathbb{C}$ by the composition of these operators. We shall show that $P_{j}(L)$ is an ambient isotopy invariant of a framed link (cf. [5] about this notation) $L$ embedded in a handlebody and that its value is in

$$
\Lambda_{n}:=\mathbb{Z}\left[q^{ \pm 1},\left\{\left(t_{j}^{(l)}\right)^{ \pm 1},\left(t_{j-1}^{(l)}\right)^{ \pm 1}, \ldots,\left(t_{-j}^{(l)}\right)^{ \pm 1}\right\}_{l=1,2, \ldots, n}\right] / \mathfrak{I}_{n}
$$

where $\mathfrak{I}_{n}$ is the ideal generated by $\left\{t_{-i}^{(l)}\left(t_{i}^{(l)}\right)^{-1}-1, t_{i_{1}}^{(l)} t_{i_{2}}^{(l)}-t_{i_{1}+\gamma}^{(l)} t_{i_{2}-\gamma}^{(l)}\right\}_{1 \leq l \leq n}$ and $n$ is the genus of the handlebody. Moreover, $P_{j}(L)$ has the following properties:

(1) $P_{j}(O)=[2 j+1]$;

Received by the editors September 30, 1991.

1991 Mathematics Subject Classification. Primary 57M25; Secondary 81R50, 20F36.

Key words and phrases. Invariant of link, framed link, handlebody, spin $j$ representation of $U_{q}(\mathfrak{s l}(2, \mathbb{C}))$, Jones polynomial, Kauffman's Dubrovnik polynomial, generalized braid group. 
(2) $P_{j}\left(\supset^{\prime}\right)=(-1)^{2 j} q^{2 j(j+1)} P_{j}(\cup)$, $P_{j}\left({ }^{\circ} \mathrm{C}\right)=(-1)^{2 j} q^{-2 j(j+1)} P_{j}(\mathrm{U})$.

Ignoring the parameters $\left\{t_{i}^{(l)}\right\}_{i, l}$ related to the embedding of a link into a handlebody, by the definition of $P_{j}(L)$, we have invariants of links in $S^{3}$. Especially, in the case of $j=\frac{1}{2}$, we shall observe that our invariant is a certain extension of the Jones polynomial $V_{L}(s) \in \mathbb{Z}\left[s^{ \pm 1 / 2}\right]$. Namely, put

$$
\widetilde{P}(L ; q):=\frac{\left(-q^{3 / 2}\right)^{-w(D)}}{[2]} P_{1 / 2}(L)
$$

then we have

$$
\widetilde{P}\left(L ; s^{1 / 2}\right)=V_{L}(s) \text { for an oriented link } L \text { in } S^{3} .
$$

Similarly, in the case of $j=1$, put

$$
\widehat{P}(\bar{L} ; q):=\frac{1}{[3]} P_{1}(\bar{L}),
$$

and let $\mathscr{D}_{\bar{L}}(a, z) \in \mathbb{Z}\left[a^{ \pm 1}, z^{ \pm 1}\right]$ be Kauffman's Dobrouvnik polynomial, then we have

$$
\widehat{P}(\bar{L} ; q)=\mathscr{D}_{\bar{L}}\left(q^{4}, q^{2}-q^{-2}\right) \text { for an unoriented link } \bar{L} \text { in } S^{3} .
$$

Moreover, as an application, we can obtain a linear representation of the generalized braid group associated with the Lie algebras of types $B$ and $C$ by applying the operators used in constructing an invariant to tangles in a solid torus.

\section{1. $U_{q}(\mathfrak{s l}(2, \mathbb{C}))$ AND ITS SPIN $j$ REPRESENTATION}

In this section, we summarize the basic properties derived from $U_{q}(\mathfrak{s l}(2, \mathbb{C}))$ and its spin $j$ representation. Let us recall that $U_{q}(\mathfrak{s l}(2, \mathbb{C}))$, the quantized universal enveloping algebra of Lie algebra $\mathfrak{s l}(2, \mathbb{C})$ in the sense of Drinfel'd [2] and Jimbo [3], is the associative $\mathbb{C}$-algebra generated by $\left\{1, k^{ \pm 1}, X^{+}, X^{-}\right\}$ with relations

$$
k X^{ \pm} k^{-1}=q^{ \pm 1} X^{ \pm}, \quad X^{+} X^{-}-X^{-} X^{+}=\frac{k^{2}-k^{-2}}{q-q^{-1}},
$$

where $q \in \mathbb{C} \backslash\{0, \pm 1\}$.

The finite-dimensional irreducible representations of $U_{q}(\mathfrak{s l}(2, \mathbb{C}))$ are parametrized by half-integers $j=0, \frac{1}{2}, 1, \frac{3}{2}, \ldots[8,10]$. This representation $\left(\varrho_{j}, V_{j}\right)$ is called the spin $j$ representation of $U_{q}(\mathfrak{s l}(2, \mathbb{C}))$, where $\operatorname{dim}_{\mathbb{C}} V_{j}=2 j+$ 1. The actions of the generators of $U_{q}(\mathfrak{s l}(2, \mathbb{C}))$ with respect to the basis $\left\{e_{m}^{j}\right\}_{-j \leq m \leq j}$ of $V_{j}$ is given as

$$
\begin{aligned}
\varrho_{j}\left(X^{ \pm}\right) e_{m}^{j} & =([j \mp m][j \pm m+1])^{1 / 2} e_{m \pm 1}^{j}, \\
\varrho_{j}(k) e_{m}^{j} & =q^{m} e_{m}^{j}
\end{aligned}
$$

where $[n]:=\left(q^{n}-q^{-n}\right) /\left(q-q^{-1}\right)$. 
It is well known that there exist an invertible element $R$ in the completed tensor product $U_{q}(\mathfrak{s l}(2, \mathbb{C})) \hat{\otimes} U_{q}(\mathfrak{s l}(2, \mathbb{C}))$, which is called the universal $R$ matrix, such that $\check{R}^{j_{1} j_{2}}:=T \circ\left(\left(\varrho_{j_{1}} \otimes \varrho_{j_{2}}\right) R\right)$ satisfies the Yang-Baxter equation

$$
\begin{aligned}
\left(\check{R}^{j_{1} j_{2}}\right. & \left.\otimes \mathbf{I d}_{V_{j_{3}}}\right) \circ\left(\mathbf{I d}_{V_{j_{2}}} \otimes \check{R}^{j_{1} j_{3}}\right) \circ\left(\check{R}^{j_{2} j_{3}} \otimes \mathbf{I d}_{V_{j_{1}}}\right) \\
= & \left(\mathbf{I d}_{V_{j_{1}}} \otimes \check{R}^{j_{2} j_{3}}\right) \circ\left(\check{R}^{j_{1} j_{3}} \otimes \mathbf{I d}_{V_{j_{2}}}\right) \circ\left(\mathbf{I d}_{V_{j_{3}}} \otimes \check{R}^{j_{1} j_{2}}\right)
\end{aligned}
$$

where $T$ is defined by

$$
T\left(e_{m_{1}}^{j_{1}} \otimes e_{m 2}^{j_{2}}\right)=e_{m_{2}}^{j_{2}} \otimes e_{m_{1}}^{j_{1}}
$$

for the basis of $V_{j_{1}} \otimes V_{j_{2}}[2,3]$. We can express $\check{R}^{j_{1} j_{2}}$ explicitly with respect to the basis of $V_{j_{1}} \otimes V_{j_{2}}$ as $[7,11]$

$$
\check{R}^{j_{1} j_{2}}\left(e_{m_{1}}^{j_{1}} \otimes e_{m_{2}}^{j_{2}}\right)=\sum_{n \geq 0}\left(R^{j_{1} j_{2}}\right)_{m_{1}, m_{2}}^{m_{1}+n, m_{2}-n}\left(e_{m_{2}-n}^{j_{2}} \otimes e_{m_{1}+n}^{j_{1}}\right) ;
$$

here the nonzero element $\left(R^{j_{1} j_{2}}\right)_{m_{1}, m_{2}}^{m_{1}+n, m_{2}-n}$ is given by

$$
\begin{aligned}
\left(R^{j_{1} j_{2}}\right)_{m_{1}, m_{2}}^{m_{1}+n, m_{2}-n} \\
:=\frac{\left(1-q^{-2}\right)^{n}}{[n] !}\left(\frac{\left[j_{1}-m_{1}\right] !\left[j_{1}+m_{1}+n\right] !\left[j_{2}+m_{2}\right] !\left[j_{2}-m_{2}+n\right] !}{\left[j_{1}-m_{1}-n\right] !\left[j_{1}+m_{1}\right] !\left[j_{2}+m_{2}-n\right] !\left[j_{2}-m_{2}\right] !}\right)^{1 / 2} \\
\quad \times q^{n\left(m_{1}-m_{2}+2 n\right)+2\left(m_{1}+n\right)\left(m_{2}-n\right)}
\end{aligned}
$$

where $[n] !:=[n][n-1] \cdots[1],[0] !:=1$. Moreover

$$
\left(\check{R}^{j_{1} j_{2}^{-1}}\right)_{m_{1}, m_{2}}^{m_{2}+n, m_{1}-n}=(-1)^{n} q^{-\left(2 m_{2}+n\right)\left(2 m_{1}-n\right)+n}\left(R^{j_{1} j_{2}}\right)_{m_{2}, m_{1}}^{m_{2}+n, m_{1}-n}
$$

where $\left(\check{R}^{\left(j_{1} j_{2}^{-1}\right.}\right)_{m_{1}, m_{2}}^{m_{2}+n, m_{1}-n}$ is a nonzero matrix element for $\left(\check{R}^{j_{1} j_{2}}\right)^{-1}$.

If $q$ is not a root of unity then the tensor representation $V_{j_{1}} \otimes V_{j_{2}}$ is decomposable into the direct sum of irreducible representations [3], that is,

$$
V_{j_{1}} \otimes V_{j_{2}}=\bigoplus_{\substack{j_{1}-j_{2} \mid \leq i \leq j_{1}+j_{2} \\ j_{1}+j_{2}+i \in \mathbb{Z}}} V_{i}
$$

The projection map $Y_{i}^{j_{1} j_{2}}: V_{j_{1}} \otimes V_{j_{2}} \rightarrow V_{i}$ is a $U_{q}(\mathfrak{s l}(2, \mathbb{C}))$-module homomorphism and is uniquely determined up to scalar multiple. Moreover, put

$$
Y_{j_{1} j_{2}}^{i}:={ }^{t} Y_{i}^{j_{1} j_{2}}: V_{i} \rightarrow V_{j_{1}} \otimes V_{j_{2}} .
$$

Then we have the following orthogonality relation between $Y_{i}^{j_{1} j_{2}}$ and $Y_{j_{1} j_{2}}^{i}$ with an appropriate normalization [7, 9]

$$
\sum_{\substack{j_{1}-j_{2} \mid \leq i \leq j_{1}+j_{2} \\ j_{1}+j_{2}+i \in \mathbb{Z}}} Y_{j_{1} j_{2}}^{i} \circ Y_{i}^{j_{1} j_{2}}=\operatorname{Id}_{V_{j_{1}} \otimes V_{j_{2}}}
$$

$$
Y_{k}^{j_{1} j_{2}} \circ Y_{j_{1} j_{2}}^{l}=\delta_{k, l} \operatorname{Id}_{V_{k}} .
$$

Let $X_{i}^{j_{1} j_{2}}:=Y_{j_{1} j_{2}}^{i} \circ Y_{i}^{j_{1} j_{2}}: V_{j_{1}} \otimes V_{j_{2}} \rightarrow V_{j_{2}} \otimes V_{j_{1}}$. Then we have a spectral decomposition of $\check{R}^{j_{1} j_{2}}$ as [4]

$$
\left(\check{R}^{j_{1} j_{2}}\right)^{ \pm 1}=\sum_{\substack{j_{1}-j_{2} \mid \leq i<j_{1}+j_{2} \\ j_{1}+j_{2}+i \in \mathbb{Z}}}(-1)^{j_{1}+j_{2}+i} q^{ \pm\left\{\xi(i)-\xi\left(j_{1}\right)-\xi\left(j_{2}\right)\right\} / 2} X_{i}^{j_{1} j_{2}}
$$

where $\xi(i)=2 i(i+1)$. 


\section{CONSTRUCTION OF INVARIANTS}

In this section, we construct invariants of links in a way analogous to that of Turaev [12].

Let $\Sigma_{n}$ be a disk with $n$ holes and $I=[0,1]$. We regard a handlebody of genus $n$ as $\Sigma_{n} \times I$. Suppose $\Sigma_{n}$ is embedded in $\mathbb{R}^{2}$ and the coordinate of the center of $\Sigma_{n}$ coincides $(0,0)$. For convenience, we assume the holes $H_{1}, H_{2}, \ldots, H_{n}$ of $\Sigma_{n}$ are arranged as shown in Figure 2.1 and the coordinates of their centers are $\left(h_{1}, 0\right),\left(h_{2}, 0\right), \ldots,\left(h_{n}, 0\right)$, respectively. Namely,

$$
\begin{aligned}
& H_{i}=\left\{(x, y) \mid\left(x-h_{i}\right)^{2}+y^{2} \leq \varepsilon^{2}\right\} \text { for } 1 \leq i \leq n, \\
& \Sigma_{n}=\left\{(x, y) \mid x^{2}+y^{2} \leq r^{2}\right\}-\bigcup_{i=1}^{n}\left\{\text { Int } H_{i}\right\}
\end{aligned}
$$

where $\varepsilon$ and $r$ are the radii of $H_{i}$ and $\Sigma_{n}$, respectively.

Let $D$ be a link diagram in Int $\Sigma_{n}$ that represents a framed link $L$ in $\Sigma_{n} \times I$. Here we assume that the diagram $D$ is taken such that the linking number of $D$ and its push-off coincides with the given framing on $L$. Such a diagram is called a good diagram in terms of Kirby-Melvin [7]. Furthermore, we assume that the diagram $D$ is taken to be generic with respect to the height function $\left.x\right|_{D}$ (or to be in general position in terms of [12]).

A point of $D$ is called critical if it is the point in which the tangent line is parallel to the $y$-axis or the self-crossing point of $D$ (Figure 2.2).

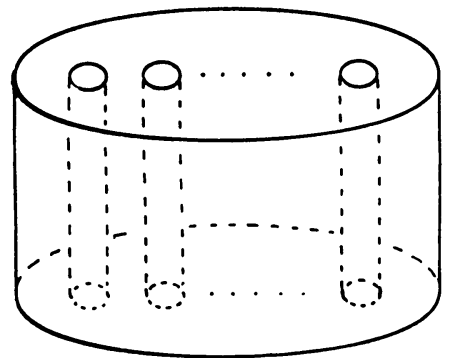

$\Sigma_{n} \times I$

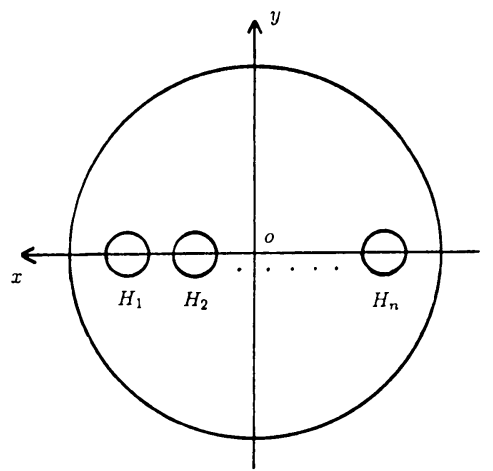

$\Sigma_{n} \times\{0\}$

Figure 2.1

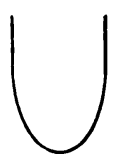

(i)

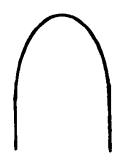

(ii)

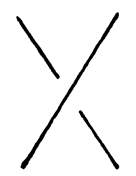

(i)

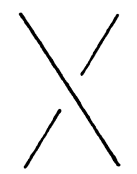

(ii)

(1)

(2)

FIGURE 2.2 
Let $d$ be the number of critical points of $D$. We draw $d+n+1$ parallel lines $\left\{x=\alpha_{i}\right\}_{1 \leq i \leq d+n+1}$ satisfying the following conditions: (1) $-r=\alpha_{1}<$ $\alpha_{2}<\cdots<\alpha_{d+n+1}=r$, (2) each open strip $\alpha_{i}<x<\alpha_{i+1}(i=1,2, \ldots, d+n)$ either contains exactly one critical point of $D$ or does not contain critical points of $D,(3)$ there exists $i$ such that $\alpha_{i}<h_{l}<\alpha_{i+1}$ for all $l=1,2, \ldots, n$, (4) none of these lines $\left\{x=\alpha_{i}\right\}$ contains a critical point of $D$.

With each line $x=\alpha_{i}, i=1,2, \ldots, d+n$, we associate a complex vector space $W^{i}:=V^{\otimes k}$ where $V$ is the representation space of the spin $j$ representation of $U_{q}(\mathfrak{s l}(2, \mathbb{C}))$ and $k$ is the number of points in $\left\{x \mid x=\alpha_{i}\right\} \cap D$. In particular, if $k=0$ then we define $W^{i}:=\mathbb{C}$.

With each strip $\alpha_{i}<x<\alpha_{i+1}$ we associate a linear operator $f_{i}: W^{i} \rightarrow W^{i+1}$ in the following way.

Let $\left\{e_{j}, e_{j-1}, \ldots, e_{-j}\right\}$ be the basis of $V$. Assume $q \in \mathbb{C}$ is not a root of unity. With the critical points depicted in Figure 2.2 we associate the following operators respectively:

(1) (i) $c:=\sqrt{[2 j+1]} Y_{j j}^{0}: \mathbb{C} \rightarrow V \otimes V$,

$$
c(1)=\sum_{i=-j}^{j}(-1)^{j-i} q^{i} e_{i} \otimes e_{-i}
$$

(ii) $a:=\sqrt{[2 j+1]} Y_{0}^{j j}: V \otimes V \rightarrow \mathbb{C}$, $a\left(e_{k} \otimes e_{l}\right)=(-1)^{j-k} q^{k} \delta_{k,-l}$.

(2) (i) $\check{R}:=\check{R}^{j j}: V \otimes V \rightarrow V \otimes V$;

(ii) $\check{R}^{-1}:=\left(\check{R}^{j j}\right)^{-1}: V \otimes V \rightarrow V \otimes V$

where $Y_{j j}^{0}, Y_{0}^{j j}$, and $\check{R}^{j j}$ are defined as in $\S 1$ with $j_{1}=j_{2}=j$. With the strand parallel to the $x$-axis we associate the identity operator $\operatorname{Id}_{V}$. Then, for the strip containing a critical point we define a linear operator $f_{i}: W^{i} \rightarrow W^{i+1}$ by

$$
f_{i}:=\mathbf{I d}_{V} \otimes \cdots \otimes \mathbf{I d}_{V} \otimes f \otimes \mathbf{I d}_{V} \otimes \cdots \otimes \mathbf{I d}_{V}
$$

where $f \in\left\{a, c, \check{R}, \check{R}^{-1}\right\}$.

Furthermore, for the strip $\alpha_{i}<h_{l}<\alpha_{i+1}$, with the strand across the segment $\left\{\left(h_{l}, y\right) \mid y \leq 0\right\} \cap \Sigma_{n}$ we associate $\operatorname{Id}_{V}$ and with the strand across the segment $\left\{\left(h_{l}, y\right) \mid y \geq 0\right\} \cap \Sigma_{n}$ we associate the operator

$$
\theta_{l}: V \rightarrow V, \quad \theta_{l}\left(e_{i}\right)=q^{x_{i}^{(l)}} t_{i}^{(l)} e_{i} \text { for } i=j, j-1, \ldots,-j
$$

where we assume that $x_{i}^{(l)} \in \mathbb{Z}$ and $t_{i}^{(l)} \in \mathbb{C}^{\times}$satisfy

$$
\begin{aligned}
& x_{-i}^{(l)}=-x_{i}^{(l)}, \quad x_{i_{1}}^{(l)}+x_{i_{2}}^{(l)}=x_{i_{1}+\gamma}^{(l)}+x_{i_{2}-\gamma}^{(l)}, \\
& t_{-i}^{(l)}=\left(t_{i}^{(l)}\right)^{-1}, \quad t_{i_{1}}^{(l)} t_{i_{2}}^{(l)}=t_{i_{1}+\gamma}^{(l)} t_{i_{2}-\gamma}^{(l)}
\end{aligned}
$$

for $0 \leq \gamma \leq \min \left(j-i_{1}, j+i_{2}\right)$, respectively. Then, for the strip $\alpha_{i}<h_{l}<\alpha_{i+1}$ (see Figure 2.3 on the next page) we define a linear operator $f_{i}: W^{i} \rightarrow W^{i+1}$ by

$$
f_{i}:=\underbrace{\mathbf{I d}_{V} \otimes \cdots \otimes \mathbf{I d}_{V}}_{u \text { times }} \otimes \underbrace{\theta_{l} \otimes \cdots \otimes \theta_{l}}_{v \text { times }} .
$$

Since $W^{i}=W^{d+n+1}=\mathbb{C}$, we have a linear operator

$$
P_{D}^{\chi_{n}}:=f_{d+n} \circ f_{d+n-1} \circ \cdots \circ f_{1}: \mathbb{C} \rightarrow \mathbb{C}
$$




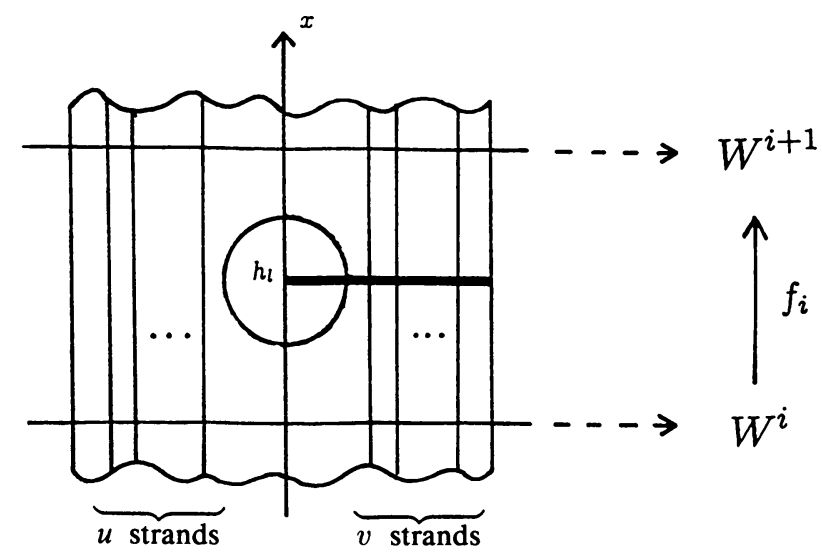

FIGURE 2.3

where $\chi_{n}:=\left\{x_{j}^{(1)}, \ldots, x_{-j}^{(1)}, x_{j}^{(2)}, \ldots, x_{-j}^{(n)}\right\}$. Suppose $q$ and $t_{i}^{(l)}(-j \leq i \leq$ $j, 1 \leq l \leq n)$ are indeterminates of $\mathbb{C}$, then, by definition, $P_{D}^{\chi_{n}}(1)$ has its value in

$$
\Lambda_{n}:=\mathbb{Z}\left[q^{ \pm 1},\left\{\left(t_{j}^{(l)}\right)^{ \pm 1},\left(t_{j-1}^{(l)}\right)^{ \pm 1}, \ldots,\left(t_{-j}^{(l)}\right)^{ \pm 1}\right\}_{l=1,2, \ldots, n}\right] / \mathfrak{I}_{n}
$$

where $\mathfrak{I}_{n}$ is the ideal generated by $\left\{t_{-i}^{(l)}\left(t_{i}^{(l)}\right)^{-1}-1, t_{i_{1}}^{(l)} t_{i_{2}}^{(l)}-t_{i_{1}+\gamma}^{(l)} t_{i_{2}-\gamma}^{(l)}\right\}_{1 \leq l \leq n}$.

We assert that $P_{D}^{\chi_{n}}(1)$ is an invariant of links in a handlebody as follows.

Theorem 2.1. Let $\chi_{n}=\left\{x_{j}^{(1)}, \ldots, x_{-j}^{(1)}, x_{j}^{(2)}, \ldots, x_{-j}^{(n)}\right\} \subset \mathbb{Z}$ be fixed. Then $P_{j}(L):=P_{D}^{\chi_{n}}(1) \in \Lambda_{n}$ is an ambient isotopy invariant of a framed link $L$ embedded in a handlebody of genus $n$ and has the following properties:

(1) $P_{j}(\bigcirc)=[2 j+1]$

(2) $P_{j}\left(\zeta^{\prime}\right)=(-1)^{2 j} q^{2 j(j+1)} P_{j}(\cup)$, $P_{j}\left({ }^{\circ} \mathrm{G}\right)=(-1)^{2 j} q^{-2 j(j+1)} P_{j}(\cup)$.

Before proving Theorem 2.1, we note the relationships among the operators. They will give the proof of the first half of Theorem 2.1.

Lemma 2.1. (1) $\left(\operatorname{Id}_{V} \otimes a\right) \circ\left(c \otimes \operatorname{Id}_{V}\right)=\operatorname{Id}_{V}=\left(a \otimes \operatorname{Id}_{V}\right) \circ\left(\operatorname{Id}_{V} \otimes c\right)$.

(2) $\left(\operatorname{Id}_{V} \otimes a\right) \circ\left(\check{R} \otimes \operatorname{Id}_{V}\right)=\left(a \otimes \operatorname{Id}_{V}\right) \circ\left(\operatorname{Id}_{V} \otimes \check{R}^{-1}\right)$, $\left(\mathbf{I d}_{V} \otimes a\right) \circ\left(\check{R}^{-1} \otimes \mathbf{I d}_{V}\right)=\left(a \otimes \mathbf{I d}_{V}\right) \circ\left(\operatorname{Id}_{V} \otimes \check{R}\right)$.

Lemma 2.2. (1) $\left(\theta_{l} \otimes \theta_{l}\right) \circ c=c$.

(2) $a \circ\left(\theta_{l} \otimes \theta_{l}\right)=a$.

(3) $\check{R} \circ\left(\theta_{l} \otimes \theta_{l}\right)=\left(\theta_{l} \otimes \theta_{l}\right) \circ \check{R}$, $\check{R}^{-1} \circ\left(\theta_{l} \otimes \theta_{l}\right)=\left(\theta_{l} \otimes \theta_{l}\right) \circ \check{R}^{-1}$.

The proofs of Lemmas 2.1 and 2.2 are given by the direct calculations from the definitions of the operators.

Proof of Theorem 2.1. As is shown in [9, 12, 13], to prove the first half of the theorem, it is sufficient to verify that each operator corresponding to the diagrams in Figures 2.4 and 2.5 is invariant under the associated modification illustrated in the figures. In fact, they are assured by Lemmas 2.1 and 2.2 and the properties of the $R$-matrix. 

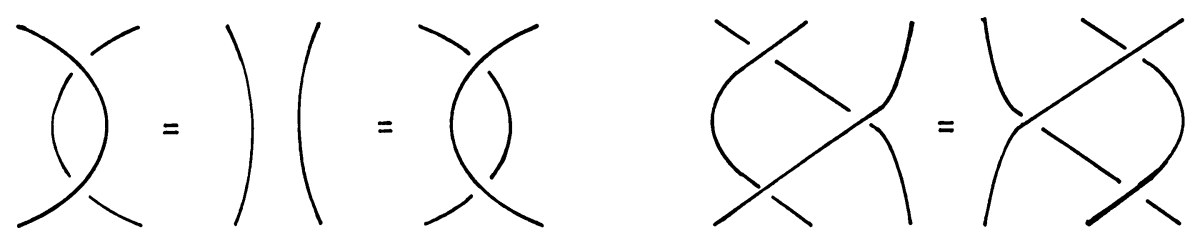

$\eta=1=\emptyset$
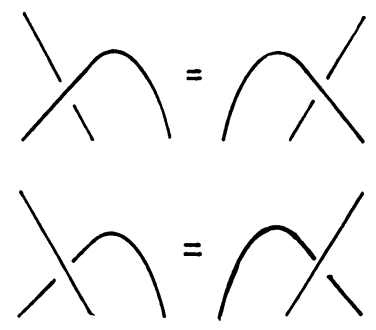

FIGURE 2.4
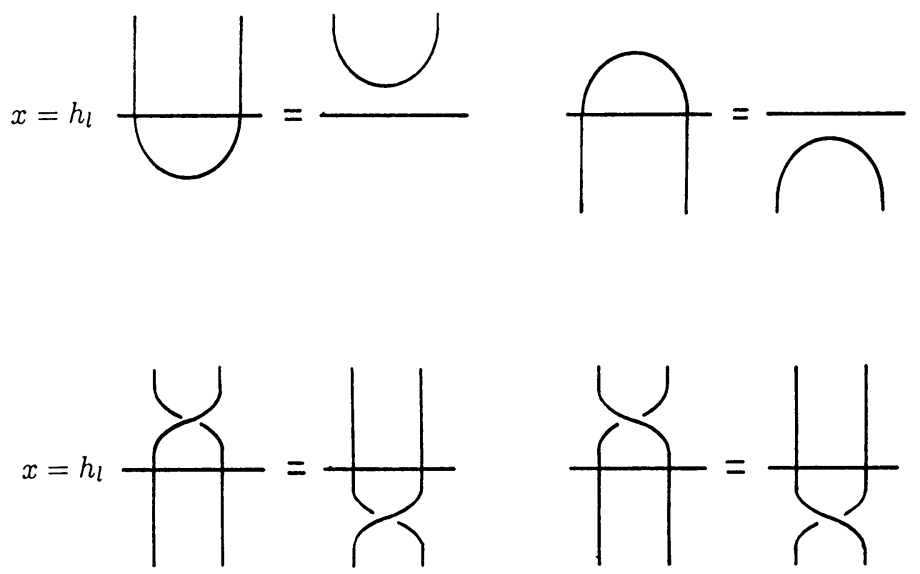

FIGURE 2.5

The properties of $P_{j}(L)$ can be easily checked as follows:

(1) By definition,

$$
P_{j}(\bigcirc)=a \circ c(1)=\sum_{i=-j}^{j} q^{2 i}=[2 j+1] .
$$

(2) By (1.4), (1.5), (1.6), and the definition of $c$,

$$
\begin{aligned}
\check{R}^{-1} \circ c & =\sqrt{[2 j+1]} \check{R}^{-1} \circ Y_{j j}^{0} \\
& =\sqrt{[2 j+1]}(-1)^{2 j} q^{2 j(j+1)} Y_{j j}^{0}=(-1)^{2 j} q^{2 j(j+1)} c .
\end{aligned}
$$

This implies the first formula. The second formula is also obtained in a similar way. 
Remark. It is clear that if $L=L_{1} \cup L_{2}$ is a split link in a solid torus then $P_{j}(L)=P_{j}\left(L_{1}\right) \cdot P_{j}\left(L_{2}\right)$.

\section{EXAMPLES}

3.1. The case of $j=\frac{1}{2}$.

Corollary 3.1. For an oriented link $L$ in a handlebody of genus $n$, put

$$
\widetilde{P}(L):=\frac{\left(-q^{3 / 2}\right)^{-w(D)}}{[2]} P_{1 / 2}(L)
$$

where $D$ is a diagram of $L$ and $w(D)$ is the writhe of $D$. Then $\widetilde{P}(L)$ is an ambient isotopy invariant of $L$ such that

(1) $\widetilde{P}(\bigcirc)=1$;

(2) $q^{2} \widetilde{P}(\nwarrow)-q^{-2} \widetilde{P}\left(\nearrow^{n}\right)=\left(q^{-1}-q\right) \widetilde{P}(\nearrow)$.

Proof. (2) is obtained by $(1.4),(1.5)$, and (1.6). The other assertions are clear.

Thus, by the definition of the invariant, we have

Corollary 3.2. Let $\chi_{n}=\{0, \ldots, 0\}$ and $\widetilde{P}(L ; q):=\widetilde{P}(L)$. If $t_{i}^{(k)}=1$ for $i=$ $-\frac{1}{2}, \frac{1}{2}$ and $k=1,2, \ldots, n$, then $\widetilde{P}\left(L ; s^{1 / 2}\right)=V_{L}(s)$ where $V_{L}(s) \in \mathbb{Z}\left[s^{ \pm 1 / 2}\right]$ is the Jones polynomial of an oriented link $L$ in $S^{3}$.

3.2. The case of $j=1$. Similarly, we obtain the following corollaries.

Corollary 3.3. For an unoriented link $\bar{L}$ in a handlebody of genus $n$, put

$$
\widehat{P}(\bar{L}):=\frac{1}{[3]} P_{1}(\bar{L})
$$

Then $\widehat{P}(\bar{L})$ is a regular isotopy invariant of $\bar{L}$ such that

(1) $\widehat{P}(\bigcirc)=1$;

(2) $\widehat{P}(Y)-\widehat{P}(\bigvee)=\left(q^{2}-q^{-2}\right)[\widehat{P}()()-\widehat{P}(\asymp)]$;

(3) $\widehat{P}\left(\partial^{\prime}\right)=q^{4} \widehat{P}(\cup), \widehat{P}(\circlearrowleft)=q^{-4} \widehat{P}(\cup)$.

Remark. Hence $\left(q^{4}\right)^{-w(D)} \widehat{P}(\bar{L})$ is an ambient isotopy invariant for an oriented link $L$ in a handlebody when $\widehat{P}(\bar{L})$ is defined on oriented link diagrams by forgetting the orientation.

Corollary 3.4. Let $\chi_{n}=\{0, \ldots, 0\}$ and $\widehat{P}(\bar{L} ; q):=\widehat{P}(\bar{L})$. If $t_{i}^{(k)}=1$ for $i=-1,0,1$ and $k=1,2, \ldots, n$, then

$$
\widehat{P}(\bar{L} ; q)=\mathscr{D}_{\bar{L}}\left(q^{4}, q^{2}-q^{-2}\right)
$$

where $\mathscr{D}_{\bar{L}}(a, z) \in \mathbb{Z}\left[a^{ \pm 1}, z^{ \pm 1}\right]$ is Kauffman's Dubrovnik polynomial for an unoriented link $\bar{L}$ in $S^{3}$.

\section{A REPRESENTATION OF THE GENERALIZED BRAID GROUP ASSOCIATED WITH THE LIE ALGEBRAS OF TYPES B AND C}

Let us recall the definition of the generalized braid group due to Brieskorn [1]. Let $\mathscr{H}_{s}$ be a hyperplane in $\mathbb{R}^{n}$. Let $W$ be a linear transformation group of 

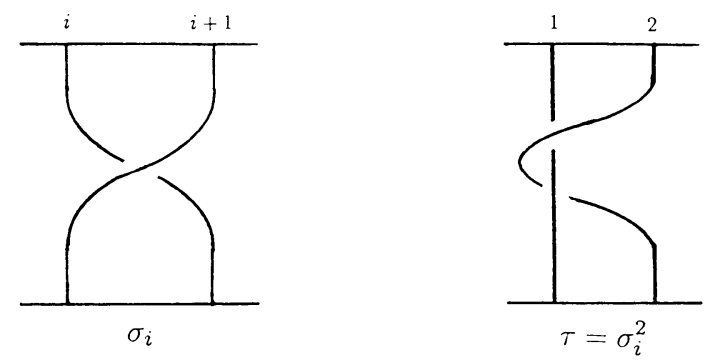

Figure 4.1

$\mathbb{R}^{n}$ generated by reflections with respect to $\mathscr{H}_{s}$ 's. We denote by $s$ the reflection with respect to $\mathscr{H}_{s}$. For a connected component of $\mathbb{R}^{n}-\bigcup_{s} \mathscr{H}_{s}$, there exists a set $\left\{\mathscr{H}_{s}\right\}_{s \in S}$ such that $(W, S)$ becomes a Coxeter system; namely, $W$ has a presentation with a generating set $S$ and a relation $\left(s s^{\prime}\right)^{m\left(s, s^{\prime}\right)}=1$ where $m\left(s, s^{\prime}\right)$ is the order of $s s^{\prime}$ and $m(s, s)=1$. Let $\mathscr{H}_{s}^{\mathbb{C}}$ be the complexification of $\mathscr{H}_{s}$ in $\mathbb{C}^{n}$. The action of $W$ is naturally extended to $\mathbb{C}^{n}-\bigcup_{s} \mathscr{H}_{s} \mathbb{C}$. In [1] Brieskorn defined the generalized braid group as the fundamental group $\pi_{1}\left(\left(\mathbb{C}^{n}-\bigcup_{s} \mathscr{H}_{s}^{\mathbb{C}}\right) / W, *\right)$ and he proved that the generalized braid group associated with $W$ has a presentation with generators $\left\{g_{s}\right\}_{s \in S}$ and a relation

$$
\left(g_{s} g_{t}\right)^{m(s, t)}=\left(g_{t} g_{s}\right)^{m(s, t)} .
$$

By investigating the root systems of the Lie algebras of types B and C, we can show that the generalized braid group $G$ obtained from the Coxeter group associated with these Lie algebras has a presentation with the generators $\left\{h, g_{2}, g_{3}, \ldots, g_{n}\right\}$ and the relations

$$
\begin{aligned}
& g_{i} g_{i+1} g_{i}=g_{i+1} g_{i} g_{i+1} \quad \text { for } i=2,3, \ldots, n \text {, } \\
& g_{i} g_{j}=g_{j} g_{i} \quad \text { for } \quad|i-j| \geq 2 \text {, } \\
& h g_{i}=g_{i} h \quad \text { for } i=3,4, \ldots, n, \\
& h g_{2} h g_{2}=g_{2} h g_{2} h \text {. }
\end{aligned}
$$

Let $B_{n+1}$ be the $(n+1)$-string braid group with the generators $\left\{\sigma_{1}, \sigma_{2}, \ldots\right.$, $\left.\sigma_{n}\right\}$ and the relations

$$
\begin{aligned}
& \sigma_{i} \sigma_{i+1} \sigma_{i}=\sigma_{i+1} \sigma_{i} \sigma_{i+1} \text { for } i=1,2, \ldots, n \text {, } \\
& \sigma_{i} \sigma_{j}=\sigma_{j} \sigma_{i} \quad \text { for } \quad|i-j| \geq 2 \text {. }
\end{aligned}
$$

By replacing the generator $\sigma_{1}$ with $\tau:=\sigma_{1}^{2}$, we can define the subgroup $B_{n+1}^{1} \subset$ $B_{n+1}$. Namely, any element of $B_{n+1}^{1}$ has the fixed first string (Figure 4.1). We can verify that there is an isomorphism $B_{n+1}^{1} \rightarrow G$ that transforms $\tau$ to $h$ and $\sigma_{i}$ to $g_{i}$ for $i=2,3, \ldots, n$.

Now we embed the elements of $B_{n+1}^{1}$ in a solid torus $\Sigma_{1} \times I$ by regarding the fixed first string as $H_{1} \times I$ as in Figure 4.2. We consider the diagrams of these embedded elements of $B_{n+1}^{1}$. The diagrams corresponding to the generators of $B_{n+1}^{1}$ are illustrated in Figure 4.3, where $\times$ indicates the fixed first string. 

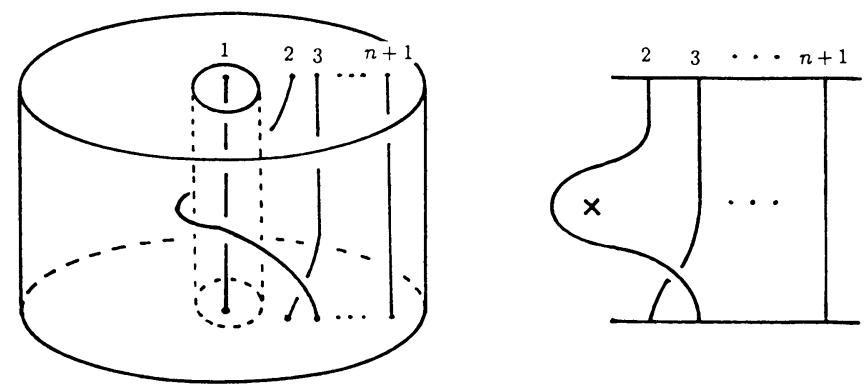

FIGURE 4.2
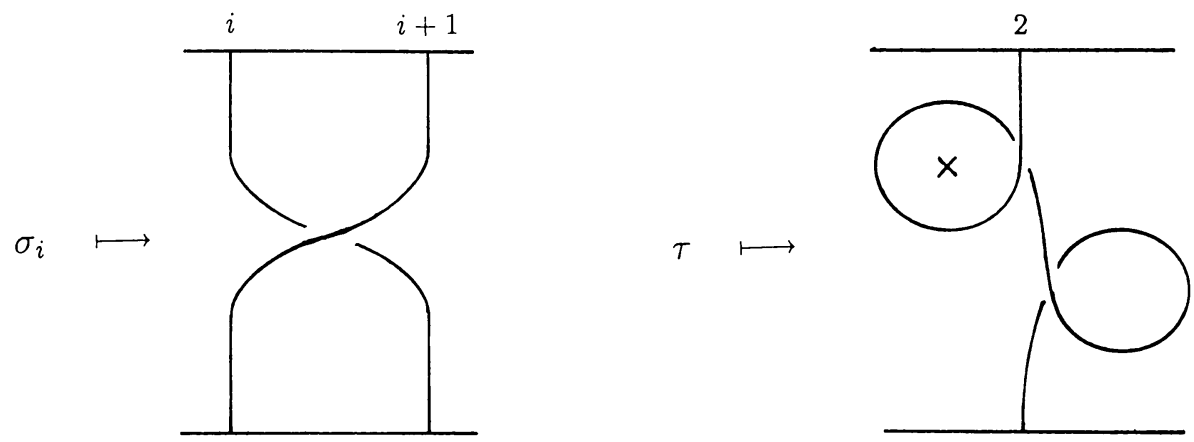

FIGURE 4.3

Then we can define the linear operators associated with the elements of $B_{n+1}^{1} \cong G$, via the diagrams mentioned above, by means of the operators defined in $\S 2$. Denote by $X_{i}$ and $Y$ the operators corresponding to $\sigma_{i}$ and $\tau$, respectively. Then we obtain the following result.

Theorem 4.1. Let $N=(2 j+1)^{n}$. The correspondence of $h \mapsto Y$ and $g_{i} \mapsto X_{i}$ for $i=2,3, \ldots, n$ gives rise to a linear representation of $G$

$$
B_{n+1}^{1} \rightarrow \mathrm{GL}_{N}\left(\Lambda_{1}\right)
$$

where

$$
\Lambda_{1}=\mathbb{Z}\left[q^{ \pm 1}, t_{j}^{ \pm 1}, t_{j-1}^{ \pm 1}, \ldots, t_{-j}^{ \pm 1}\right] / \mathcal{I}_{1}
$$

and $\mathfrak{I}_{1}$ is the ideal generated by

$$
\left\{t_{i} t_{-i}-1, t_{i_{1}} t_{i_{2}}-t_{i_{1}+\gamma} t_{i_{2}-\gamma}\right\} \quad \text { for }-j \leq i \leq j, 0 \leq \gamma \leq \min \left(j-i_{1}, j+i_{2}\right) .
$$

Proof. It is easily checked that the operators corresponding to $X_{i}$ and $Y$ satisfy the relations of $G$ since our operators are invariant under regular isotopy. For example, the relation $Y X_{2} Y X_{2}=X_{2} Y X_{2} Y$ follows from Figure 4.4. 


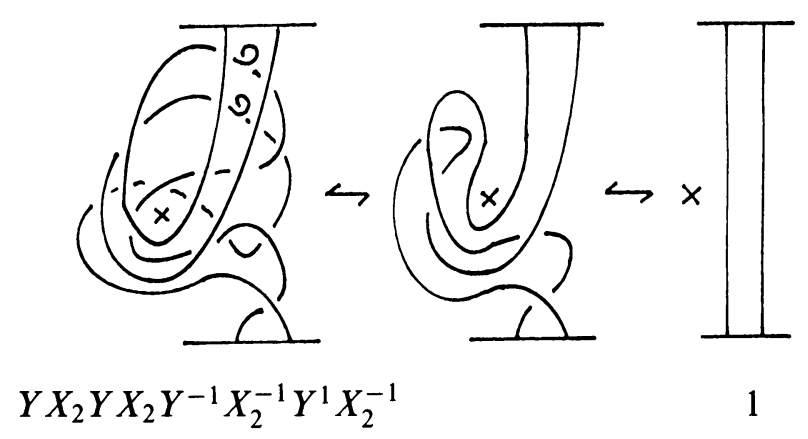

FIGURE 4.4

\section{ACKNOWLEDGMENT}

I would like to thank Professor T. Kohno for his helpful suggestions.

\section{REFERENCES}

1. E. Brieskorn, Die Fundamentalgruppe des Raumes der regulären Orbits einer endlichen komplexen Spiegelungsgruppe, Invent. Math. 12 (1971), 57-61.

2. V. G. Drinfel'd, Quantum groups, Proc. Internat. Congr. Math. (Berkeley), vol. 1, Amer. Math. Soc., Providence, RI, 1986, pp. 798-826.

3. M. Jimbo, $A$ q-difference analogue of $U(\mathfrak{g})$ and the Yang-Baxter equation, Lett. Math. Phys. 10 (1985), 63-69.

4. Math. Phys. 11 (1986), 247-252.

5. L. H. Kauffman, An invariant of regular isotopy, Trans. Amer. Math. Soc. 318 (1990), 417-471.

6. R. Kirby and P. Melvin, The 3-manifold invariants of Witten and Reshetykhin-Turaev for sl(2, C), Invent. Math. 105 (1991), 473-545.

7. A. N. Kirillov and N. Yu. Reshetikhin, Representations of the algebra $U_{q}(\mathfrak{s l}(2))$, q-orthogonal polynomials and invariants of links, Infinite dimensional Lie algebras and groups (V. G. Kac, ed.), World Scientific, Singapore, 1989, pp. 285-342.

8. G. Lustig, Quantum deformations of certain simple modules over enveloping algebras, Adv. in Math. 70 (1988), 237-249.

9. H. R. Morton and P. Strickland, Jones polynomial invariants for knots and satellites, Math. Proc. Cambridge Philos. Soc. 109 (1991), 83-103.

10. M. Rosso, Représentations irréductibles de dimension finie du q-analogue de l'algèbre envloppante d'une algèbre de Lie, C. R. Acad. Sci. Paris Sér. I Math. 305 (1987), 587-590.

11. N. Yu. Reshetikhin and V. G. Turaev, Ribbon graphs and their invariants derived from quantum groups, Comm. Math. Phys. 127 (1990), 1-26.

12. V. G. Turaev, The Conway and Kauffman modules of solid torus with an appendix of the operator invariants of tangles, LOMI preprint E-6-88 (1988).

13. D. N. Yetter, Markov algebra, Contemp. Math., vol. 78, Amer. Math. Soc., Providence, RI, 1988, pp. $705-730$.

Department of Mathematics, Kyushu University, Fukuoka 812, Japan

E-mail address: nakabo@math.sci.kyushu-u.ac.jp 